crois rapide, et dans une certaine mesure, tangible à l'observation humaine.

"Ce chapitre de l'évolution souterraine révèle d'emblée, comme un abime brusquement ouvert, le sort futur de notre planète entière: audouble jeu de la multiplication tectonique des cassures et de leur approfondissement érosif, la dessication finira par intéresser les mers aussi bien que les continents.

"Quand l'intérieur de la terre engloutira et retiendra plus d'eau qu'il n'en restituera. ce phénomène de la dessication s'accélerera effroyablement vers la généralisation des saharas terrestres.

"C'est donc le dessèchement aboutissant à la disette d'eau, qui s'annonce comme la plus probable, la plus normale, la plus rapide de toutes les éventualités».

Que pouvons-nous donc pour parer à ces futurs évènements? - Le mieux, pour le moment, est de chercher à conserver cette eau si précieuse et qui tend à s'enfouir sous nos pas. M. Martel conseille pour cela deux remèdes : d'abord le reboisement, et la reconstitution des sols montagneux qui tendent à retenir l'eau au profit de l'industrie et des besoins naturels de l'homme et à enrayer l'infiltration; ensuite l'extension des explorations souterraines qui font connaître les meilleurs moyens d'utiliser les réceptacles d'eau douce. Car, sans eau, le monde ne peut vivre; sans eau, la force motrice ferait défaut à son tour, puisque déjà le gisements de combustibles (houille, pétrole, etc.) s'épuisent sous nos yeux et sans pouvoir être retrouvés. L'homme finira bien évidemment par utiliser la chaleur solaire, désormais seule force vive dont il disposera. Reste à savoir, pour notre consolation, si les générations futures survivront elles-mêmes à de tels désastres et si elles n'auront pas disparu avant que ces phénomènes se soient montrés dans leur plus cruelle activité ! Cela est très possible.

Quoi qu'il en soit de ces perspectives plus philosophiques que scientifiques, laissons le globe évoluer comme la nature le lui commande. Profitons, aux moments présents, des forces naturelles qu'il nous distribue aux uns et aux autres, si profusément, mais ne soyons pas dissipateurs. Toute évolution dit changement d'état : la terre, comme les êtres qu'elle fait vivre et développe, ne saurait échapper à la loi qui mène le monde vers une fin que nous ignorerons toujours.

Jean Escard, Ingénieur civil.

\section{LA RICHESSE DU RHONE et son utilisation}

Cet article est la reproduction d'un rapport présenté le 27 mars 1908 à la Sociélé d'économic politaque et d'économie sociale de Lyon, et dine communication fate le 3 aout 1908 a congres de ClermontForrand de l'Associalion francaise pour lavancement des sciences, par M. René TavenNier, ingénieur en chef des Ponts et chaussées, par M. René TavenNier, ingénieur en chef des Ponts et Chaussées,
directeur de l'Office des T'ansports, oftice organisé par les Chambres de Commerce du sud-est de la France.

\section{I. - EXPOSÉ, COUP D'CEIL RÉTROSPECTIF}

Le Rhône est une richesse exposée, comme toutes les autres, à subir des fluctuations qui, pour n'être pas aussi soudaines que celles des valeurs cotées en Bourse, n'en sont pas moins considérables. A n'envisager que la consistance même de la richesse du Rhône, - les débits très variables, suivant les saisons, qui caractérisent ce qu'on appelle son "régime", - il semble que ces fluctuations ont l'ampleur, difficile à mesurer, des pérıodes géologı́ues, des progressions ou des régressions glaciaires. Si l'on considère, non pas le "Iégime" du fleuve, mais les ulilısations diverses auxquelles al se prète, on peut dire que chargue jour l'évaluation de la richesse du Rhòne prend des aspects inattendus.

Le problème de son aménagement " intégral " n'est pas résolu, nı près de l'être ; mais le seul fait qu'il soit aujourdhui posé est un signe suffisamment caractéristirue de l'époque actuelle. Le temps n'est plus où l'eau du grand fleuve pouvart être considérée par les économistes comme une matière premıère sans valeur, aussi inépuisable que l'air, et dont chacun pouvait user à sa convenance, pourvu qu'll ne gĉnat perscnne. Ces aspect convenalt à l'éporque. qui vient à peine de prendre fin, des emplois individuels et des entreprises isolées. Les riverains qui s'abreuvaient au Rhône, ou s'en servaıent pour arroser leurs cultures, les navigateurs qui se hasardaient sur ses flots rapides, ne se préocicupaient, pas plus il y a cent ans qu'à l'époque gauloise, d'aménagement " intégral ".

Avec les programmes d'ensemble visant, soit l'amélioration du Rhône navigable, sout la création de grands canaux d'irrigation, et surtout avec les mstallations hydro-électriques dont Jonage a été le premier type, s'ouvre véritablement une ère nouvelle. Longtemps avant que la richesse du Rhône devint réellement insuffisante, on a commencé à se Ja disputer. C'est surtcut à Lyon, véritable métroprole du Rhòne, que se sont agitées toutes ses conceptions nouvelles : et la Société d'Eiconomie politique a été, à diverses reprises, le champ clos de discussions dont le souvenir n'est point effacé.

Lie moment est venu, peut-être, non pas de réveiller de vielles querelles, mais de montrer pilutôt dans quelle mesure l'évolution récente de la "houille blanche " en a déplacé le terrain. On n'est pas près de s'entendre sur la manière d'utiliser le Rhône, ni surtout d'avoir groupé les capitaux nécessaires à la réalisation d'un programme général d'amélioration qui alle de la mer à Lyon, et même à Genève. Mais ciependant, plus que jamais, les vues d'ensemble sont nécessaires pour faire rentrer harmoniquement dans un mème cadre ces vastes entreprises qui viennent aujourd'hui, d'un seul coup, mettre en valeur des tronçons importants du fleuve.

Vous connaissez les principales élapes des grandes transformations modernes.

C'est d'abord l'initiative lyonnaise qui mène à bien, vers le milieu du siècle dernier, la double entreprise du canal et du Port-Saint-Louis. Le Rhône étart désormais raccorclé à la mer ; sa barre était tournée. Aujourd'hui, l'initiative marseillaise entreprend un canal grandiose, qui ne doit pas coûter moins de 71 millions, pour rattacher le Rhône au grand port méditerranéen. Cette entreprise, dont on a si vivement contesté l'utilité, aura ce double effet de faire contribuer le Rihône au développement de Narseille, et de permettre en même temps à l'industrie marseillaise d'employer directement, sans intermédiaires, ses puissantes ressources à la mise en faveur du fleuve. Notez, je vous prie, comme un indice significatif de l'évolution économique générale, qu'entre les Chambres de commerce de Marseille et de Liyon, une parfaite entente a été conclue au sujet d'entreprises jadis rivales. Saint-Louis n'a pas nui au développement de Marseille ; il a seulement satisfait des besoins nouveaux, dont on peut chiffrer l'importance en comparant son tonnage effectif à celui du port de Marseille (346.500 tonnes contre 6.488.067 tonnes en 1902). Dans un pays qui n'y est pas habitué, comme l'Amérique, la navigation du Rhône a fait éclore une ville, un port, et même une gare dont le trafic est bien le seul cadeau que la Compagnie P.-L.-M. ait, avec bonne grâce, consenti à recevoir d'une voie navigable. Quant aux craintes que peut faire naitre l'établissement 
du canal de Marseille pour l'avenir de Saint-Louis, M. Guérard (et personne en la matière n'est plus autorisé que lui) les estime chimériques. Il pense que " le canal de Marseillé " au Rhône apportera à la navigation du Rhône les éléments " de trafic qui, jusquà présent, lui ont fait presque complè" tement defaut et que Saint-Louis, port auxlliaire de Mar"seille, en profitera".

En même temps que le canal de Marseille, celui beaucoup mons coûteux de celle va s'exécuter aussi, de telle sortc que les batelıers dì Rhône auront à leur dispositıon, dans quelques années, trois ports qui remplaceront avantageusement ceux d'Arles et d'Aigues-Mortes utilisés au moyen-âge.

Voici donci qu'à l'embouchure du Rhône l'harmonie s'est fate entre tous les intérêts. Grâce à l'initiatıve persévérante des Chambres de commerce de Marselle et de Cette prolongeant et complétant, à un demi-siècle de distance, l'inrtiative ale M. Peut, le fleuve va être complètement et rationnellement aménagé.

Dans les parties du Rhône à fortes pentes, le problème élaıt plus compliqué ; il a paru longtemps insoluble. M. l'Inspecteur général Vallée rapporte, dans un ouvrage publié en 1843 (1), que "d'après l'opinion dominante, le Rhône était " un fleuve indomptable dont il y avait peu de chose à tirer " et qu'sl fallait abandonner à lui-même ". En effet, pendant le longues années, le Rhône a résisté victorieusement aux efforts des ingénieurs qui ne faisaient, passez-moi l'expression, que le "taquiner"; s'attaquant isolement aux mauvais massages, ils ne les corrigaient sur un point que pour les voir reparaitre un peu plus loin. Il était rélservé à notre regretté et éminent collègue, M. Girardon, de coordonner les Afforts de ses prédécesseurs, de créer une méthode d'amélioration continue, partout admirée et suivie, venant fixer, après de nombreux tâtonnements, l'art de l'ingénieur nyydraulicien. Le Rhône terrible, obligé d'employer ses forces à l'entretien de son propre chenal, était " domestiqué ".

Assurément, ce programme ne réalise qu'un aménagement partiel ef limité. La puissance du Rhône ne reçoit qu'une utilisation restreinte. Mais personne ne pouvait soupçonner, il y a trente ans, qu'il y eût un autre parti à en tirer. L'œuvre des ingénieurs, menée à bien par M. Girardon, reste digne d'admiration.

Mais voici que, par un revirement soudain, les pentes oxcessives du Rhône, qui étaient jusçu'ici une cause d'inférıorité, deviennent son principal élément de richesse, celui sur lequel on doit le plus légitimement compter pour l'aménagement de certains tronçons.

A l'amont de Lyyon, le canal industriel de Jonage offe à la navigation un superbe bief de 18 kilomètres, le plus beau de toute la vallée, et le seul qui n'ai rien coûté aux finances publiques. C'est encore à l'initiative hardie de quelques Lyonnais, de véritables précurseurs, qu'on doit cette grande œuvre. Et voici qu'à la frontière suisse on projette un aménagement plus grandiose encore (2) qui transformera le tronçon le plus innavigable du Rhône, de la frontière à Génissiat, en un second bief également magnifique de plus de 20 kilnmètres de longueur. Sans doute, Messieurs, ne s'agit-il là que d'un projet. Mais les forces du haut Rhône ont une telle valeur, elles ont été l'objet de compétitions si vives, qu'on ne saurait considérer comme bien éloignée l'heure de la réalisation.

L'idée, jadis préconisée par M. Vallée, de rattacher le lac de Genève aux voies navigables de France, vient à son heure au moment où l'on se préocicupe sérieusement en Suisse d'ouvrir à la batellerie du Rhin l'accès du lac de Con'stance et même, par le lac de Neufchâtel, celui du Léman (3).

(1) L. Vallée. Du Rhône et du lac de Genève. - Paris 1843.

(2) Projet d'utilisation des Forces motrices dı Rhône drescé nar

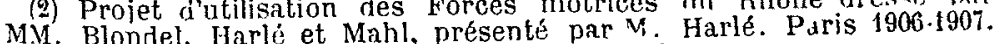
Voir La Houille Blanche, janvier et mars 1007.

(3) Importance de la Suisse comme centre du développement des voies (3) Importance de la Suisse comme centre du développement des voies
navigables de l'Europe Centrale par Rud. Gelpke, ingénieur à Bàle.
Mais, je ne veux pas, Messleurs, après avoir tracé a grands traits le tableau des principales i.ranslormations du Rhòno à l'époque actuelle, me laiser aller plus longtemps à ces vues générales. Vous attendez, de mos, sans doute, quelques indcations plus précises. Si vous le voulez, je maltachera dabord à caractérıser le "régme " du Rhòne, cest-a-dire les délits varıbles qui concourent, avec sa pente, a mesurer sa richesse hydraulique. Ensuite, je passerai successivement on revue, en m'en tenant au point de vue économique, les diverses utilisations dont cette richesse est susceptible : lalimentation des villes, lirrigation, la navigation, la créatron de forces motrices. Jaura rempli ma tache si cet examen vous suggère, sans que j'y mette trop du mien, quolques conclusions qui nauront pas la netteté dun projet d'ingénleur, mass qui suffront, peut-ètre, je n'ai pas l'aulre ambition, à provorguer des études et des recherches nouvelles.

\section{II. - LA RICHESSE DU RHONE. - NOTIONS HYDROLOGIQUES}

Deux causes bien connues concourent à rélgulariser le débıt du Rhóne à sa sortie du lac le Genève :

$1^{\circ}$ Limportance, dans le bassm du Rhóne suisse, dos neiges et des glaciers;

$2^{\circ}$ Linfluence du lac lui-mème conctionnant comme résel voir.

Le bassin alımentaire du Rlone, à son entrée dans lc Léman, mesure 5.220 kilomètres carris, dont 933 kllometres de glaciers. C'est une proportion tout à fart exceptionnelle, qui est assez loın d'être atteinte lans le bassin similaire du Haut-Rhin. Par l'Arve, le Rhónc recoll encore, à sa sortic du lac de Genève, les écoulements du Mont-Bianc. Jusqua Lyon, ou tout au moins jusquä lembonchure de l'inn, lo régime du Rhône est donc franchement glaciaire.

Les neiges et les glaciers constituent aulant diommagasnements automaliques qui se forment en hiver pour disparaître au printemps. Les cours d'eau qui subissent les eflels périodiques de ces emmagasinements sont exposés a des variations presque aussi fortes que les cours d'eau directement alimentés par les pluies ou des sources. Leur grande. supériorité industrielle tient à deux causes : ils ont des eaux abondantes en été, au moment de l'étiage des autres cours d'eau, et, de plus, leurs débits, variant régulıèrement avec les saisons, suivant une loi de périodicité qui ne change pas beaucoup d'une année à l'autre, peuvent être plus comr modément ef plus complètement utilisés.

Le lac de Genève, fonctionnant comme un réservoir naturel, ninflue pas autant que les glaciers sur les variations saisonnières du Rhône. Il a plutôt pour effet d'atténuer les variations bruscues et extremes, soit des crues, soit des étiages. Mais son rôle peut être élarọi.

En 1811, M. Vallée, inspecteur général des Ponts ot Chaussées, avait proposé d accroître artificiellement la puissance de régularisation du Rhóne. Il voulait mettre, suivant, sa formule expressive, "les eaux du Léman dans les mains de l'homme" et, par ce moyen. améliorer les conditions de navigabilité, porter remède aux inondations et créer des forces motrices (1).

ll proposait pour cela : $1^{\circ}$ de jeter l'Arve dans le lac do Geneve ; $2^{\circ}$ de faire varier Je niveau du lac entre les cotes limites de $0 \mathrm{~m} .45$ et $2 \mathrm{~m}$. 25 en llessous du repère de la pierre de Niton, soit un écart de $1 \mathrm{~m} .80$ nermettant d'emmagasiner, la superficie du lac étant de 578 kilomètres carrés, plus d'un milliard de mètres cubes, c'est-à-dire de foumir au Rhône, pendant 115 jours, un débit supplémnetaire de 110 mètres cubes à la seconde.

Imprimerie Atar, Corraterie 12, Genève 1907. Voir La Houille Blanche, mail 1908.

(1) Valrée. - Des eaux, des travaux publics et du barrage de Genève, Paris 1859 . 
Ces vues, trop harlles prour lépoque, nabouturent pas, malgré lencouragement que leur acicorda Napolćon III, mais clles vulgarisèrent une idée juste et féconde. Ramenées à des proportions plus modestes par une appréciation plus exacte des interets ruverains, elles inspirèrent la Convention intercantonale du 17 décemirre 1884 relative à la correchon du Rhône et à la régularisation de l'écoulement des eaux du lac Léman. Par celte convention, les coles limites extrèmes du niveau du lac furent fixées à $1 \mathrm{~m}$. 30 et $1 \mathrm{~m}$. 90 au dessous du repere de la pierre de Nilon, sout un écart de $0 \mathrm{~m}$. 60 correspondant à une tranche d'cau de 316.800 .000 mètres cubes.

Lientreprise, réduite au tiers de ce qu'avait prévu Vallée, fit sentir ses résultats. Diaprès une notice (1) de M. Van Muyden, ingénieur à Lausanne, à laquelle j'emprunte ces chiffres, le jeu de la retenue a permis daccumuler dans le lac, pendant les mors d'avril, mar, juin et jullet, daprès la moyenne délcennale résultant des statistiques des années 1890 à 1900,325 millions de mètres cubes qui ont été rendus à l'émissaire pendant les mois de janvier, février, mars, septembre, octobre, novembre el décembre. Le niveau mojen réel du lac, plus rapproché de sa limite supérıeure que de sa limite inférieure, a été de $1 \mathrm{~m}$. 50 au-dessous du repère de la pierre de Niton.

L'étiage conventıonnel, qui était avant la régulatıon de 70 à 80 mètros cubcs, semble avoir été fixé depuls à 100 mètres cubes au sortir du lac, 120 mètres cubes à l'aval du confluent de l'Arve.

Cetle pierre de Niton, qui a servi de base à la régularisation du lac de Genève, a des origmes ancıennes que je me reprocherais de ne pas vous signaler en passant, y trouvant l'acicasion de semer un peu de poésıe dans l'ariulutél des chifleses.

Il existail jadis, dans le port de Genève, raconte Vallée, plusieurs rochers consacrés à Niton, nom que se clonnait Neptune quand ll frécuentait la mer de Genève. Comme ces rochers formaient écueil, on los fit sauter. On en conserva cependant un sur lequel on fixa un repère, préposant ainsi lantique divinité à la surveillance du nıveau dont dépend lalimentation du fleuve.

Franchement glaciaire à som entrée en France, le Rhòne se grossil peu à peu daffluents qui viennent modifier profondément son régime. Les affluents de rive droite : l'Am, dont la crue a dépassé, en 1836, celle du Rhòne Iui-même, la Saône, l'Ardèche, ont un régime tout diflérent. Les affluents de rive gauche alimentés par les Alpes : le Fier, l'Isère et la Durance, ont un régime mixte, où les écoulements glaciaires manifestent une influence de moins en moins accentuée.

Naturellement, je ne veux insister sur ces apercus d hydrologle que dans la mesure nécessaire pour fare linventaire de la richesse du Rihone. Mais il ne suffit pas aujourd'hui, comme autrefois, de connaitre approximativement les débits extrèmes : les étiages pour s'en accomoder et les crues pour s'on garer. Avco les grands aménagements hydro-électriques les eaux moyennes deviennent utilisables. Il faut en étudier les variations. Deux débits nous serviront à caractériser la valeur industrielle du fleuve : le débit d'étiage normal, et le débit moyen c'est-à-dire le débit sur lequel on peut compter, année moyenne, pendant six mois par an.

Les débits caractéristiques ainsi clénnis peuvent être déterminés pour une sérıe d'années à l'aide d'observations régulierement poursuivies; mais ils ne doivent pas être considérés comme immuables. Des causes, dont les unes sont naturelles et les autres artificielles, peuvent les modifier assez profondément.

Ia " glaciologie ", science toute nouvelle, signale presque partout le recul des glaciers. Lies écoulements glaciaires

(1) Van Muyden. - Le rígıme du lac Léman et de sès affiuents.
Genève 1901 . subissent de ce fait des majorations factices qui constituent une véritable dépense de " capptal ".

Par contre, les régulations artificielles par des réservoırs. petits ou grands sont partout éludiées avec ardeur dans lebut d'acicroitre la continuité de la production de houlle. blanche. Sans doute, les facilıtés, encore insuffisamment uhllisées, qu'offre le Léman pour réaliser d'un seul coup des. emmagasmements puissants, ne se rencontrent-elles pas ailleurs ? Mais si, dans chaque bassin, on multiplie les pethles. réserves, elles finiront par infuencer sérieusement les débils d'étiage des affluents et, par contre-coup, celui du fleuve lü même.

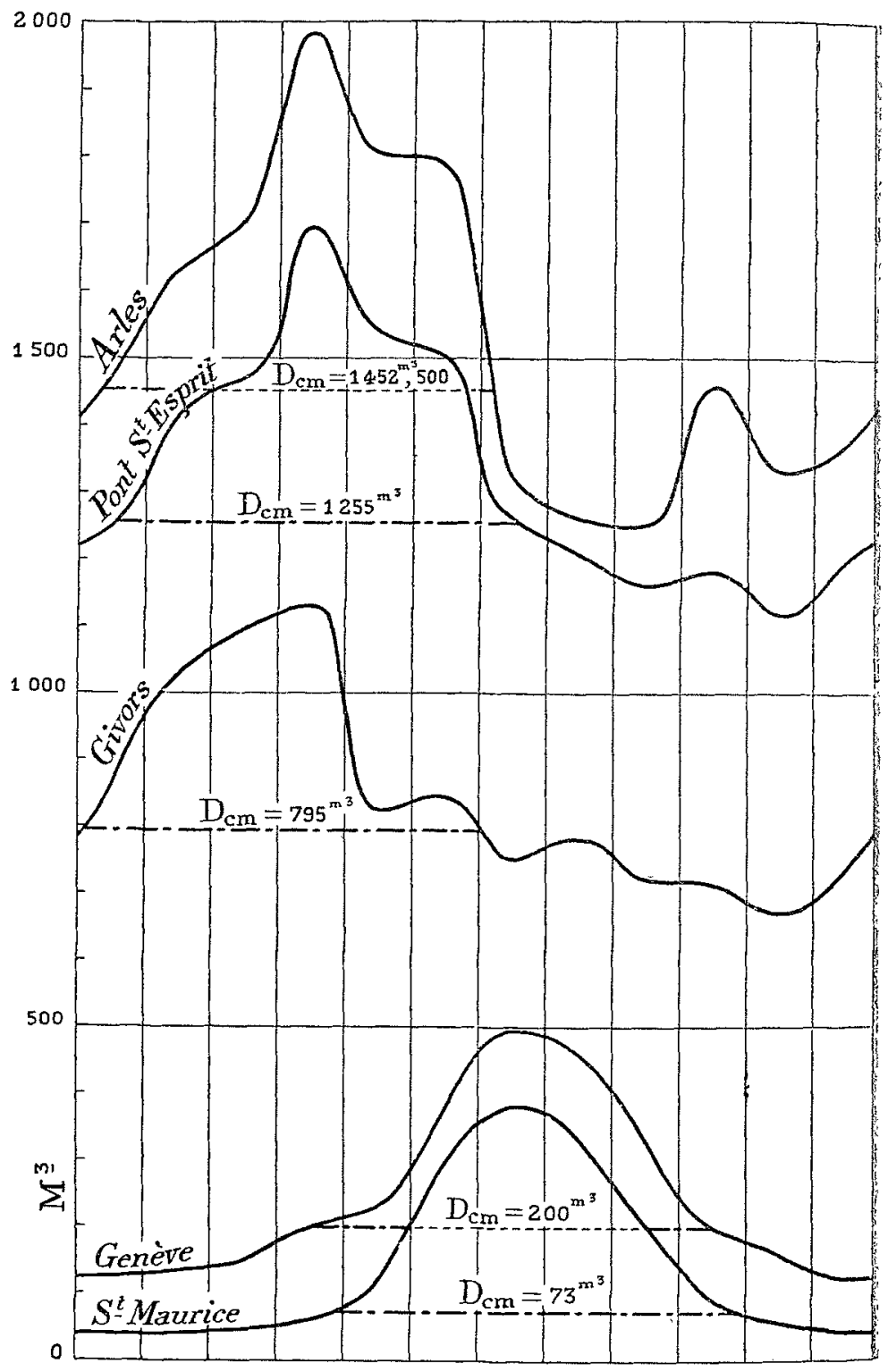

J. F. M. A. M. J. J. A. S. O. N. D.

FIG. 1. - Graphique des débits moyens mensuels du Rhône en divers points de son cours $\left({ }^{*}\right)$.

Les graphiques ci-joints fig. (1) représentent les moyennes mensuelles des débits du Rhône à son entrée dans le Lémanr à sa sortie à Givors, à Pont-Saint-Esprit et à Arles.

Les moyennes mensuelles ont été calculées en prenant lest résultats moyens d'une sérıe d'années. Ce sont, peut-on dire, des moyennes au seciond degré, qui effacent les irrégularités. dues aux conditions météorologiques si variables, vous le savez, d'une année à l'autre et qui n'accusent pas non plus les crues exceptionnelles.

Ces graphiques suffisent cependant, sous leur forme simplifiée, à définir le régime" "normal " du fleuve aux diffé-

(*) D $\mathrm{cm}$ indique le débit caractérıstique moyen.

(1) Voir dans La Houille Blanche d'avril 1908 les graphiques des. débits du Rhône à la frontıère franco-suisse. 
rents points que j'ai considérés. Ils permettent également de détorminer les débits caractéristiques, ainsı que la période de l'annéle pendant laquelle ces débits caractéristıques devront normalement se produire.

Remarquez, du reste, que les écarts que l'on constate entre le régime " normal " et le régime " réel " d'une année prise a part sont d'autant plus acicusés que prédomine l'alimentation par les pluies sur lalimentation glaciarre. La fonte des neiges ef des glaciers est un phénomène dont le retour est ḋ peu près régulier. Il n'en est pas de même des pluıes, essentielloment capricieuses sous nos climats.

Ajant déterminé les débits "caractéristiques » du Rhône aux points principaux de son cours, je serai en mesure de vous donner une idée de l'ensemble de sa richesse hydraulique en plaçant sous vos yeux un profil en long schématıque comportant deux bandes, dont les largeurs mesurent les débits caractéristiques, et dont les surfaces mesurent les puissances (figure 2).

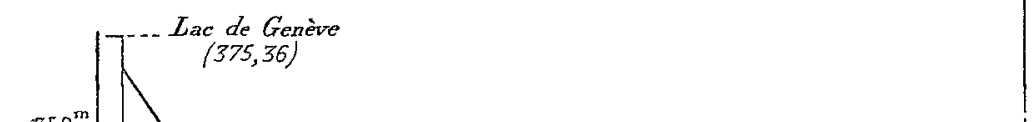
profil en long du Rhône avec figuration schéma'ique des puissances utilisables.

La patte teintee inténzure correspond au debit d'étagag. La partie teiutée comprise entre les trails exterieurs collespond aux eaux moyennes lequel je veux altirer volre altention, par une utilisation notable des ressources du Rhòne.

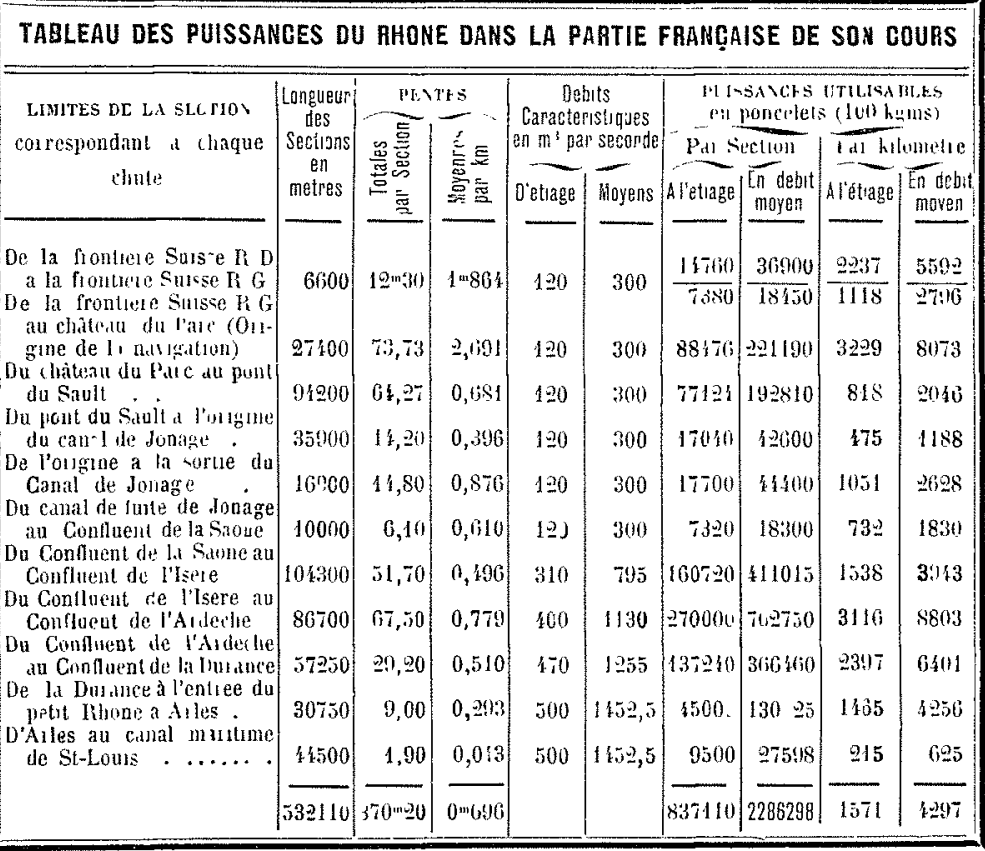

Dêconposition de la surrace du Bassin du Rhône
Bassiu du Rhône en amont de lirre........ $6004 \mathrm{Km}^{2}$ Bassin de li.Arve............ . . .. ... 1916 -

Bacsin de l'Am. . ................ . . $3512 \quad-$ Bassin des petits alfluents en amont de Lyon . 6508 BASSIN dU RHONE A I.XON ........... ...... 1096 $\mathrm{km}^{2}$ Bassin de la Saone... ..... . .. . . 28548 -

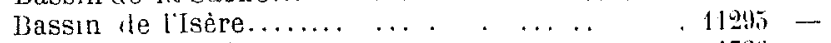
Bassin de la Drôme. . .

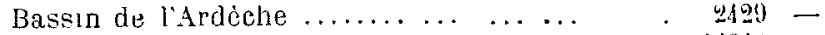
Bassin de la Durance....................... 11814 Bassin du Gardon.... .. .. .............. 2073 Bassin des petits affuent entre Lyon el la mer. $1505-$

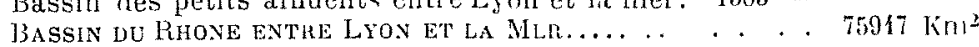

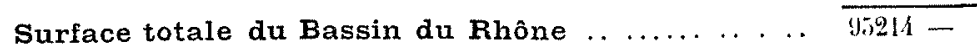

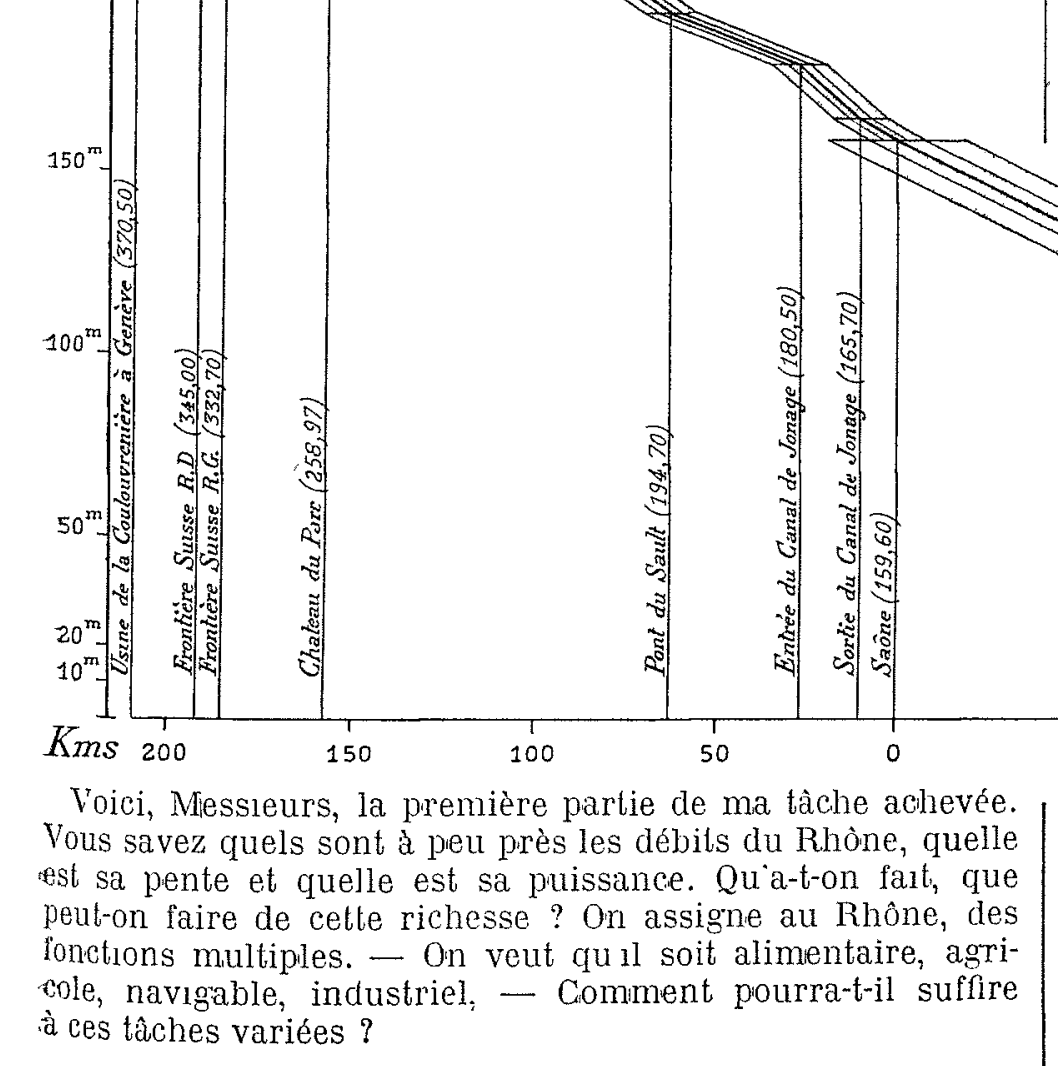

\section{III. - LES UTILISATIONS DU RHONE}

Rhône alimentaire:- Les besoins de l'alimentation et de I'hygiène profitent, en ce qui concerne au moins les populalions de la vallée, d'une sorte de privilège qui n'est pas contesté mais qui ne se traduit pas, c'est le seul point sur 
à la France entıère, on peut dıre que la richesse du Rliòne ne s'en trouvera pas sensiblement dimınuée.

Rhône agricole. - Jusquà ce jour, malgré de grands projets, les campagres ne prenneut pas direclement au Rhône pour les irrigations beaucoup plus d'eau que les villes pour leur alimentation

Le seul canal d'arrosage dérivé du Rhône présentant quelque importance est le canal de Prerrelatte. Sa portée autorisée est de 8 mètres cubes, et son pérımètre arrosable de 20.000 hectares; mass comme, en réalité, on n`a pas trouvé de propriétaires s'engageant à payer la taxe de 50 francs par hectare pour une surface supérieure à 1.500 hectares, le canal n'emprunte au Rhône, à raıson de un litre à la seconde par heclare irrigué, qu'un débit de un mètre cube et demi. C'est un véritable insuccès économique. Il liest dailleurs pas le seul. Tous les autres grands canaux modernes établis dans le bassin du Rhône, et dérivant l'eau de ses principaux affluents. sont dans une situation analogue. Je vous citerai, notamment, non lom du canal de Pierrelatte, le canal de la Bourne qu ne dérive, lui aussi, qu'un mètre cube et demi, n'uthlisant qu'une faible partie de sa dotation qur est de 7 mètres cubes. Dans le bassin de la Durance, les canaux de Gap, de Ventavon et bien d'autres encore, ont donné lleu à de graves mécomptes.

Par contre, les vieux canaux de la basse Durance ne suffisont plus à alimenter les territoires qui se disputent leurs eaux. Chaque année, lis enlèvent à la Durance la totalıté de son débit d'étiage, à peu près égal à 70 mètres cubes à la seconde.

Ces inégalités, si accusées dans la réussite, dues pour une part à des mrconstaces particulıères malheureuses, trouvent lour explıcatıon générale dans certaınes causes d inférıorité mhérentes au mode dexploitation des grands canaux. Le jérımèlre dominé lımıte étroıtement leur clientèle. La pratirque de larrosage, substituant la culture intensive à la culture extensive, exige des transformations coûteuses et lentes. I.es propriétaires qui ne peuvent y fare face assez promptemont sont ruinés par l'eau qui deval les enrichir. Dans quelques pays pauvres, tels que la région de Gap, il est arrivé que les taxes d'arrosage, perçues sous une forme anticommerciale en vertu d'engagements intangubles souscrits pour 40 ou 50 ans, ont grevé si lourdement certams fonds que ceux-ci ont réellement perdu une grande partie de leur valeur.

Malgré cet insuccès - momentané faut-il espérer - des grands canaux modernes, ll reste exact de dire (les cultures de la basse Durance en sont un exemple magnifique), que l'eau est en agriculture, toutes les fois qu'on a les moyens de la buen utiliser,et que les conditions de climat et du sol s'y prêtent, le plus actif stimulant de la production. Supposez, comme le cas se présente parfois à l'ćtranger dans les pays de grandes propriétés, que le périmètre arrosé par un cana] d'irrigation soit exploité par une seule personne, ou par une société ayant les ressources suffisantes pour réaliser toutes Jes transformations utiles, le succès financier ne serait pas douteux.

La cause d'insuccès que je viens de signaler disparaît si I'on fait de l'irrigation par des pompages. Ce système n'a rien de bien nouveau. On a songé bien souvent, pour le Rhóne notamment, à remplacer des têtes mortes très longues par des machines élévatoires à vapeur, et toujours la solution a été reconnue trop coûteuse, inapplicable aux grandes entreprises. Mais l'emploi de l'énergie hydro-électrique change aujourd'hui les conditions du probleme.

Eit je ne puis mieux yous faire sentir l'économie de cette solution nouvelle qu'en vous faisant connaitre l'aboutissement final des projets de grands canaux dérivés du Rhône, projets Dumont, projet Chambrelent, qui ont si vivement et depuis longtemps préoccupé l'opinion publique.

A la suite de nombreux remaniements, le programme définıtivement adoplé en 1897 consistait à exécuter trois. dérivations indépendantes:

$1^{\circ}$ Une dérivation de rive gauche empruntant 12 mètres. cubes à l'Isère, près de Romans;

$2^{\circ}$ Une dérivatıon de rive droite emprunlant 23 mètres cubes au Rhòne, à Cornas ;

$3^{\circ}$ Une dérivation inféricure de rive droite empruntant 12 mètres cubes au Rhône en aval du confluent de la Cèze, vers l'Ardoise.

La dépense prévue se chiffrait ainsi :

$1^{\circ}$ Canal de rive gauche..$\ldots \ldots \ldots \ldots \ldots$

$2^{\circ}$ Canal de rive droite jusçu'à Nìmes......

$3^{\circ}$ Canal de la Cèze .................... 30 millions 85 43 30

Ensemble. . .

188 milions

L'exécution devart être entreprise dès que le total des souscriptions recueillies azrait atteint cinq mètres cubes et demí pour le canal de rive gauche, dix-neuf mètres cubes pour le canal entier de rive droite, cunq mètres cubes et demı pour le canal de la Gìze.

Cette vaste entreprise a eu d'ardents apôtres : elle a paru particulièrement séduısante au moment où le Midi réclamait de l'eau, non seulement en été pour les irrigations de cultures, mais encore en hiver pour les submersions de vignes. En ajoutant à ces deux sources de revenus les produits à tirer de l'eau d'alımentalion en eau continue, que l'olk comptait vendre aux villes (notons en passant ces chiffres) quinze fois plus cher que l'on ne vendait l'uau d'arrosage aux campagnes (1.000 francs le litre à la seconde contre $63 \mathrm{fr} .50)$, Il semblaut qu'on pût arriver à joindre les deux bouts malgré que le prix de revient du mètre cube dérivé fùt, en général, plus élevé pour les canaux dérivés du Rhône que pour les autres grands canaux exécutés, pour ceux en particulier dont je viens de vous rappeler l'insuccès (1).

Messieurs, malgré beaucoup de tentatives, aucune combinaison financrère n'a paru offrir les garanties suffisantes pour qu'on pût, à aucun moment, entreprendre l'exécution de ce vaste programme.

Aujourd'hui, les conditions sont bien changées.

Le département de l'Agriculture déclare qu'il n'est guère raisonnable de songer à entreprendre de nouveaux canaux dans la vallée du Rhône, tant qu'on utilisera pas plus complè tement les canaux déjà construits. Il redoute les conséquences d'un nouvel insuccès.amplifié dans des proportions considérables. D'autre part, la réciente crise vilicole a fail sentir plus que jamais la nécessıté de substituer la polyculture à la monoculture de la vigne. Si l'on déverse l'eau du Rhône dans les plaines de Nîmes, ce ne sera pas, comme il y a dix ans, pour favoriser la pratique des submersions; bien au contraire, on cherchera à développer tous les arrosages, sauf celui de la vigne.

Et voici la combinaison qui paraît, à l'heure actuelle, rallier les suffrages. L'eau du Rhône serait pompée le plus près possible des régions à irriguer, c'est-à-dire à un endroit où elle est manifestement surabondante, au moyen de l'énergie hydro-élecitrique de la basse Durance transportée à 100 kilomètres. Les têtes mortes très coûteuses seraient supprimées. La dépense du premier établisemment serait réduite. Les pompagaes seraient exactement proportionnés aux besoius.

(1) Voici les jrix de revient de premier établissement par mètre cube dérivé :

Canaux dérivés du Rhône (montant des projets ne comprenant pas la distribution) :

1. Canal de rive gauche...... \& $500.000 \mathrm{fr}$

Ganal de rive droite....... 5.565 .000

3. Canal de la Céze............ 2500.000

Autres canaux (comprenant je réseau de distribution) :

Canal de Marseille .......... $5000.000 \mathrm{fr}$.

Canal du Verdon.............. 3.500 .000

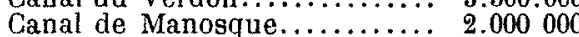

Canal de la Bourne.......... 1600.000

Ganal de Pierrelatte.......... 1.150.000 
Admettez que, pour commencer tout au moins, on limite les arrosages aux périmètres d'altitudes modérées. Admettez encore qu'on utılise pour les pompages les résidus d'énergie dinstallations hydro-électriques tirant leur principal revenu d'emplois rémunérateurs mais intermittents. Eit vous recon!nâtrez qu'on a quelques raisons de se laisser séduire par une solution qui a, par dessus tout, le mérite de pouvoir être essayée sur une petite échelle sans absorber, au début, des capitaux considérables (1)

Le département de l'Agriculture s'y montre favorable, renonçant pour le momenl à poursuivre l'exécution du projet Chambrelert.

(A suivre.)

R. TAVERNIER.

Ingénieur en chef des Ponts el Chaussées

\section{TRANSPORT D'ÉNERGIE MOUTIERS-LYON Par courant continu à 50000 volts}

Le transport d'énergie Moutiers - Lyon fonctionne depuis 1906. Le courant continu-série (système Thury) fut choisi par suite des conditions spéciales que devait remplir l'installation. Il s'agissait de transporter à 180 kilométres une puissance globale de plus de 3500

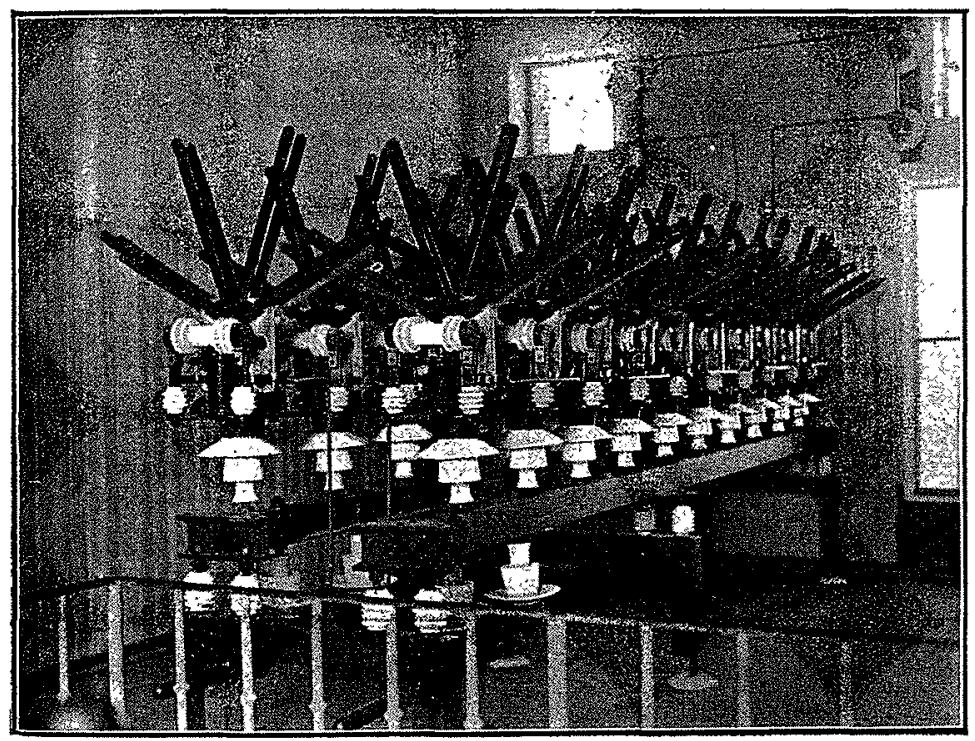

FIG. 1. - Vue d'une batterie de parafoudre à soufflage magnétique.

kilowatts, sans avoir à distribuer en cours de route aucune énergie, la région en étant sulfisammenl pouvue.

(1). A la sulte d'une communication faite le 5 août 1908, par M. Victor BolLève, membre de la Chambre de Commerce de Béziers à la sectron du Génie Cevil du Congrès de Clermont-Ferrand de l'association Francaise pour l'Avancement des Sciences, cette section a émis le rou survant:

"Considérant la situation générale de la viticulture méridionale qui constitue une des branches les plus importantes de la production nationale;

«Considérant que l'irrigation seule peut permettre la polyculture,c'esta-dure, l'introduction des cultures varićes, dans les dó,partemeris du Gard et de l'Hérault, obligés aujourd'bui par la sécheresse de leurs terres, à la culture à peu près exclusive de la vigne;

"Considérant qu'il est possible, sans nuire à la navigation du Rhône, ni à l'utilisation industrielle de sa force motrice, d'emprunter au fleuve l'eau nécessaure à l'irrigation des deux départements, en la prenant dans le bas de son cours, où le débit est plus que sufflsant pour assurer la navigation, et le plus prc̀s possible des terrains à irriguer, et l'élevant à a hauleur nécessaire par l'emploi de l'énergie électrique amenée des lieux où elle peut être économiquement produite;

Emet le vou:

Que,sans abandonner les études tendant à la solution du grand problème de lutilisation du Rhône pour la navigation et la production de la force motrice, mais en en disjoignant complèlement celles qui se rapportent à son utilisation agricole, il soit procédé d'urgence aux travaux d'irrigation du Gard et de l'Hérault par une prise deau sur le Rhône dans la partie inférieume de son cours où le débit est plus que sufflsant aux besoins de la navigation ".
La grande distance qui sépare les stations génératrice et réceptrice imposait un très haut voltage pour ne pas avoir des peries en ligne inadmissibles. Or, en alternatif, un haut voltage efficace correspondant à un plus haut voltage maximum, eut exigé un isolement très soigné, de plus, les tensions alternatives fatiguent beaucoup plus les isolants. D'autre part, le fait d'avoir à transporter à l'extrémité de la ligne toute la puissance engendrée, fit songer au système continu-série, que son unique promoteur, $M$. Thury, avait déjà réalisé dans différents pays, et dont voici, à titre d'indication, la liste chronologique.

\begin{tabular}{|c|c|c|c|c|c|c|}
\hline 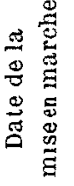 & INSTALLATION & 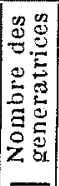 & 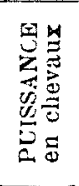 & 华 & 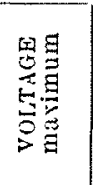 & 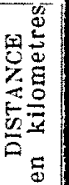 \\
\hline 1889 & $\begin{array}{c}\text { Société Acquedotto De Ferrar } \\
\text { Galliera, Gènes (Italie)......... }\end{array}$ & 18 & 1260 & 45 & 14000 & 60 \\
\hline 1891 & Service des eaux de Zug (Suisse). & 5 & 600 & 50 & 8000 & 24 \\
\hline $\begin{array}{l}1893 \\
1895\end{array}$ & $\begin{array}{l}\text { Papeteries de Biberıst (Suisse). } \\
\text { Communes du Val de Travers } \\
\text { Fleurler, Norraigue, Travers, } \\
\text { Couvet (Suisse) ............... }\end{array}$ & 2 & 370 & varialse & 6800 & 185 \\
\hline 1895 & 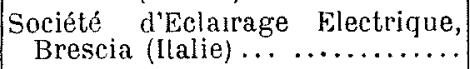 & 5 & 850 & 50 & 10500 & 26 \\
\hline 1895 & $\begin{array}{l}\text { Soclété Romande d'Electricité. } \\
\text { Forces motrices de la Grande } \\
\text { Eau, Aigle (Suisse)........... }\end{array}$ & 4 & 1000 & 50 & 14000 & 18 \\
\hline 1890 & $\begin{array}{l}\text { Usines Electriques d'Eisenburg, } \\
\text { Ikervar-Steinamanger (Hongrie) }\end{array}$ & 6 & 900 & 65 & 9000 & 65 \\
\hline 1896 & 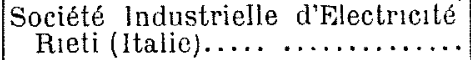 & 4 & 500 & 30 & 12000 & 30 \\
\hline 1896 & $\begin{array}{l}\text { Papeteries de la Société Yasco- } \\
\text { Belga, Renteria (Espagno)...... }\end{array}$ & 5 & 1270 & 65 & 13. $\varepsilon 0$ & 14 \\
\hline 1896 & $\begin{array}{r}\text { Communes de La Chaux-de-Fonds } \\
\text { el Du Locle (Suisse) .... ...... }\end{array}$ & 8 & 2700 & 150 & 12500 & 26 \\
\hline 1896 & $\begin{array}{l}\text { Usines Electriques d'Eisenburg. } \\
\text { lkervar-Sopron (Hongrie)....... }\end{array}$ & 4 & 600 & 40 & 10.100 & 120 \\
\hline 1899 & V. Dunand, Batoum (Russie).... & 2 & 200 & 50 & 2600 & 10 \\
\hline 1900 & Mınes de plomb, Lınarès (Espagne) & 3 & 960 & 60 & 10500 & 30 \\
\hline 1901 & $\begin{array}{c}\text { Commune de Lausanne, Lau- } \\
\text { sanne, St-Maurice (Suisse)..... }\end{array}$ & 12 & 6000 & 150 & $2 \tau 000$ & 56 \\
\hline 1905 & $\begin{array}{l}\text { Sjciété-Grenobloise de Force et } \\
\text { Tumiére,Moutiers-Lyon(France) }\end{array}$ & 16 & 6300 & 75 & 57.600 & 180 \\
\hline
\end{tabular}

Le transport d'énergie Moutiers-Lyon se fait à l'intensité constante de 75 ampères. A pleine charge, la puissance fournie aux bornes des génératrices est de $4320 \mathrm{kws}$, le voltage au départ à pleine charge est donc égal à 57600 volts.

En temps normal, et contrairement à ce qui a été dit souvent à ce sujel, aucun point de linstallation n'est mis directement à la terre. Cependant, à la station de Vaulx-en-Veliri, près de Lyon, deux voltmètres électrodynamiques sont branchés: le premier entre la terre et uu des fils de ligne, le deuxième entre la terre et le deuxième fll de ligne; ce qui revient à les mettre directementen série, leur point médiant étant à la terro. Dans ces conditions, lorsque l'isolement des deux fils de ligne est identique, chacun d'eux est porté à un potentiel maximum égal à 25300 volts, ce voltage étant pris par rapport au sol $\left(^{*}\right)$. Mais lorsque, pour une raison quelconque, l'isolement d'un des deux fils devient plus faible (et cela arrive fréquemment), le potentiel de ce fil s'abaisse, tandis que celui du deuxième fil s'élève. Dans le cas extrême où un fil se mettrait franchement à la terre. le voltmètre branché sur ce fil n'indiquerai plus rien, tandis que l'autre serait porté au maximum de tension. On voit l'inconvénient qu'il y aurait dans ce dernier cas à avoir (comme on avail eu l'intention de le faire tout d'abord), le point milieu des génératrices à la terre: un court-circuit franc se produirait alors. Il n'y a de mise directe à la terre qu'en cas de réparations sur la ligne.

Indépendamment du voltage précédent (maximum $576 \mathrm{co}$ ), et qu'on pourrait appeler le potentiel a dynamique, simplement parce qu'il est la cause même de la transmission 